\title{
Molecular Characterization and Clinical Impact of TMPRSS2-ERG Rearrangement on Prostate Cancer: Comparison between FISH and RT-PCR
}

\author{
A. Fernández-Serra, ${ }^{1}$ L. Rubio, ${ }^{1}$ A. Calatrava, ${ }^{2}$ J. Rubio-Briones, ${ }^{3}$ R. Salgado, ${ }^{4}$ R. Gil-Benso, ${ }^{5}$ \\ B. Espinet, ${ }^{4}$ Z. García-Casado, ${ }^{1}$ and J. A. López-Guerrero ${ }^{1}$ \\ ${ }^{1}$ Laboratory of Molecular Biology, Fundación Instituto Valenciano de Oncología, C/Professor Beltrán Báguena 8, \\ 46009 Valencia, Spain \\ ${ }^{2}$ Department of Pathology, Fundación Instituto Valenciano de Oncología, C/Professor Beltrán Báguena 8, 46009 Valencia, Spain \\ ${ }^{3}$ Department of Urology, Fundación Instituto Valenciano de Oncología, C/Professor Beltrán Báguena 8, 46009 Valencia, Spain \\ ${ }^{4}$ Laboratori de Citogenètica Molecular, Servei de Patologia, GRETNHE, Programa de Recerca en Càncer, IMIM, Institut de Recerca \\ del Hospital del Mar, Parc de Salut Mar, 08003 Barcelona, Spain \\ ${ }^{5}$ Department of Pathology, Universitat de Valencia Estudi General, 46010 Valencia, Spain
}

Correspondence should be addressed to J. A. López-Guerrero; jalopez@fivo.org

Received 26 December 2012; Accepted 30 April 2013

Academic Editor: Sachidanand Pandey

Copyright (C) 2013 A. Fernández-Serra et al. This is an open access article distributed under the Creative Commons Attribution License, which permits unrestricted use, distribution, and reproduction in any medium, provided the original work is properly cited.

Prostate cancer $(\mathrm{PCa})$ is a very heterogeneous disease, and there are constraints in its current diagnosis. Serum PSA levels, digital rectal examination (DRE), and histopathologic analysis often drive to overdiagnosis and overtreatment. Since 2005, the presence of the genetic rearrangement between transmembrane-serine protease gene (TMPRSS2) and the erythroblast transformation-specific (ETS) member ERG (v-ets erythroblastosis virus E26 oncogene homolog avian) has been demonstrated in almost half of PCa cases. Both FISH and RT-PCR are useful tools for detecting these rearrangements, but very few comparatives between both techniques have been published. In this study, we included FFPE tumors from 294 PCa patients treated with radical prostatectomy with more than 5 years of followup. We constructed a total of 20 tissue microarrays in order to perform break-apart and tricolor probe FISH approaches that were compared with RT-PCR, showing a concordance of $80.6 \%(P<0.001)$. The presence of TMPRSS2-ERG rearrangement was observed in $56.6 \%$ of cases. No association between TMPRSS2-ERG status and clinicopathological parameters nor biochemical progression and clinical progression free survival was found. In conclusion, this study demonstrates that both FISH and RT-PCR are useful tools in the assessment of the TMPRSS2-ERG fusion gene status in PCa patients and that this genetic feature per se lacks prognostic value.

\section{Introduction}

Prostate cancer ( $\mathrm{PCa}$ ) is a heterogeneous disease, which ranges from indolent to lethal behaviour [1]. The diagnosis may be clinically suspect based on an elevated serum prostate specific antigen (PSA) and/or abnormal digital rectal examination (DRE), the definitive diagnosis established by histopathologic examination of needle biopsy tissue. However, both PSA and DRE often lead to both overdiagnosis and overtreatment presenting limitations when differentiating between indolent and aggressive PCa [2]. In addition, biopsy is also far from being optimal because it has demonstrated a lack of sensitivity and high risk of morbidity for patients [3]. Hence, in this context, there is an increasing demand of specific biomarkers for PCa diagnosis that also provides information regarding the prognosis of the disease.

In 2005 a novel set of fusion genes were described in nearly half of the PCa cases [4] involving the $5^{\prime}$-untranslated region of TMPRSS2 (21q22) and the codifying region of some transcription factors such as ERG (21q22), ETV1 (7p21), 
and ETV4 (17q21) among others [5]. To date, there are 10 different genes involved in these genetic fusions [6]. However, TMPRSS2-ERG is the most prevalent, and more than 20 variants of the TMPRSS2-ERG fusion transcripts have been described $[6,7]$, and the exon 1 of TMPRSS2 with the exon 4 or 5 of ERG (T1E4 or T1E5, resp.) are the two most frequently involved variants [8]. The main mechanisms by which the TMPRSS2-ERG fusion genes are produced are interstitial deletion and balanced translocation [9]. Because of their specificity, detection of these fusion genes could be a valuable ancillary diagnostic tool in the early detection of PCa [10]. In fact, these rearranged genes can be detected either by fluorescence in situ hybridization (FISH), reverse transcription polymerase chain reaction (RT-PCR) techniques [7,11-14], or branched DNA (bDNA) analysis that is a very sensitive approach [15].

FISH is considered the gold standard in the detection of fusion rearrangements; the break-apart strategy is the main approach used for this propose [12]. Yoshimoto et al. developed a three-colour assay able to distinguish between the two main mechanisms of gene rearrangement for TMPRSS2-ERG, the interstitial deletion, or the reciprocal translocation [16]. In this sense, a commercial FISH assay comprising threecolor (tricolor) probes has been developed that are able to discriminate between the putative fusion gene partners and the different gene rearrangement mechanisms.

The aim of this study is to compare FISH and RT-PCR techniques in the assessment of the TMPRSS2-ERG fusion gene in a series of 294 cases of PCa and to establish the prognostic usefulness of a commercial FISH tricolor deletion probe.

\section{Material and Methods}

2.1. Case Selection. Formalin fixed and paraffin-embedded (FFPE) blocks corresponding to PCa patients were retrieved from the archives of the Biobank of the Fundación Instituto Valenciano de Oncología according to the following criteria: radical prostatectomy specimens and no history of previous treatment for $\mathrm{PCa}$ (including androgen deprivation therapy or chemotherapy prior to surgery). We obtained $294 \mathrm{PCa}$ specimens that met these criteria during the period between 1996 and 2008. All patients gave written informed consent, and the study was approved by the Ethics Committee of our institution (reference no. 2006-07). The clinical data were reviewed from the clinical records and stored in a PCa-specific database. The main characteristics and patient demographics are shown in Table 1. In addition, we also analyzed 20 samples of normal prostate tissue as controls obtained from radical cystectomies neither of which was benign prostatic hyperplasia or PCa. Haematoxylin and eosin $(\mathrm{H} \& \mathrm{E})$ stained slides were reviewed and Gleason pattern was assigned.

2.2. TMAs Construction. We constructed 19 tissue microarrays (TMAs) comprising 294 tumor samples and another TMA with 20 samples of normal prostatic tissue used as control, containing three representative nonnecrotic cores of
TABLE 1: Demographics and main clinical and pathological features of the analyzed series.

\begin{tabular}{lcc}
\hline Parameters & $n$ & $\%$ \\
\hline PSA & & \\
$\quad<10 \mathrm{ng} / \mathrm{mL}$ & 186 & 63.3 \\
$\quad 10-20 \mathrm{ng} / \mathrm{mL}$ & 66 & 22.4 \\
$\quad>20 \mathrm{ng} / \mathrm{mL}$ & 42 & 14.3 \\
Gleason-sp & & \\
$\quad \leq 6$ & 118 & 40.1 \\
7 & 139 & 47.3 \\
$\quad 8-10$ & 37 & 12.6 \\
$\mathrm{cT}$ & & \\
$\quad \leq \mathrm{cT} 2 \mathrm{~b}$ & 273 & 92.9 \\
$\quad \geq \mathrm{cT} 3 \mathrm{a}$ & 19 & 6.5 \\
$\mathrm{pT}$ & & 54.4 \\
$\quad \leq \mathrm{pT} 2$ & 160 & 45.2 \\
$\quad \geq \mathrm{pT} 3$ & 133 & \\
$\mathrm{pN}$ & & 64.6 \\
pN0 & 190 & 3.4 \\
pN $\geq 1$ & 10 & 42.7 \\
Perineural invasion & & 49.2 \\
$\quad$ Negative & 126 & \\
Positive & 145 & \\
\hline
\end{tabular}

SP: specimen, cT: clinical stage, pT: pathological stage, PSA: prostaticspecific antigen, and $\mathrm{pN}$ : pathologic stage with respect to lymph node status. *Lymphadenectomy was limited to the obturator fossa in most of the cases at the inclusion period.

each case ( $1 \mathrm{~mm}$ in diameter). TMAs were constructed using a manual tissue arrayer (Beecher instruments, Silver Spring, $\mathrm{MD})$.

2.3. Fluorescence In Situ Hybridization (FISH). TMPRSS2$E R G$ fusion status was determined by using both a breakapart assay and a triple-labelled colour commercial probe KBI-10726 (Poseidon, Kreatech Diagnostics, The Netherlands) flanking both TMPRSS2 and ERG. This probe is designed to detect the deletion between TMPRSS2 and ERG at 21q22 region but also translocations involving this region with other genes such as ETV1 or ETV4.

For the break-apart assay we used two noncommercial flanking probes (red (R) and green $(\mathrm{G})$ ) to $E R G$ to detect rearrangements affecting this gene [5]. BAC clones covering both distal and proximal regions of $E R G$ on chromosomes 21q22, CTD-2341018, and CTD-219A22, respectively, were obtained from Children's Hospital Oakland Research Institute (Oakland, CA, USA). The selection of these BAC clones was performed using the genome browser from Centre de Regulació Genòmica, Barcelona, Spain (http://davinci.crg.es/). BAC DNA isolation was performed according to Qiagen Plasmid MIDI kit protocol (Qiagen Inc., Valencia, CA) and was labelled using a nick translation kit (AbbottMolecular, Abbott Park, IL). CTD-219A22 and CTD-2341018 BACs were labelled with the Spectrum Red-dUTP (AbbottMolecular) and Spectrum Green-dUTP (AbbottMolecular), respectively. 
The cytogenetic localization of all BACs was verified by hybridization to normal metaphase spreads (G-banding with inverted DAPI).

The FISH assay was carried out on $3 \mu \mathrm{m}$ thick FFPE tissue sections. After deparaffinization, tissue sections were treated with a commercial FFPE tissue section kit (MAD-FISH-PKII, Master Diagnostica, Granada, Spain) following the manufacturer's instructions. Briefly, the slides were immersed in thiocyanate solution at $80^{\circ} \mathrm{C}$ for 30 minutes and then treated with a protease solution for 10 minutes at $37^{\circ} \mathrm{C}$. Afterwards, the tissue sections were pretreated and probes were denatured at $80^{\circ} \mathrm{C}$ for 5 minutes and hybridized overnight at $37^{\circ} \mathrm{C}$ using the hybridization System HyCrome (Euroclone S.pA., MI, Italy). Posthybridization washes were performed using a posthybridization solution (MAD-FISH-PKII, Master Diagnostica, Granada, Spain) for 2 minutes at $72^{\circ} \mathrm{C}$ and then, the slides were counterstained with $7 \mu \mathrm{L}$ of 4 , 6-diamino-2phenylindole (DAPI II, Vysis, Downers Grove, IL). Results were visualized using a ZEISS, Imager.Z1 fluorescent microscope with the AxioCam HRc camera the AxioVision 4 software (Carl ZEISS MicroImaging GmbH, Germany).

2.4. Criteria for the FISH Interpretation. The main mechanisms of the TMPRSS2-ERG rearrangement are interstitial deletion or translocation [17]. In our break-apart strategy, two yellow (Y) or R and $\mathrm{G}$ adjacent signals indicate a normal pattern, with no rearrangement in any of the two alleles. Whereas one $Y$ and one R or G signal alone or one $Y$ signal plus one $\mathrm{R}$ and one $\mathrm{G}$ separated signals indicate interstitial deletion and translocation, respectively. Thirty-eight cases of PCa were evaluated using break-apart probe in order to compare this approach with tricolor probes (Figure 1).

The Poseidon TMPRSS2-ERG FISH (21q22) Del, Break, TC (Kreatech Diagnostics, Netherlands) consists of three probes: R, G, and blue (B). A nonrearranged case would show a triplet of RGB signals; however, a translocated case would be represented by one triplet signal corresponding to the normal allele accompanied by another B and $\mathrm{G}$ fused and a separated R signal. On the contrary, an interstitial deletion would be shown by one fused triplet signal plus a B and R fused signal. Another predominant pattern consisting of one fused signal plus a B and $G$ adjacent signal was also observed, called the break pattern. Moreover, a fused RGB signal with a separated $\mathrm{B} / \mathrm{G}$ or $\mathrm{R}$ signal alone was assigned to unknown rearrangement (Figure 1).

A total of 50 nuclei of each of the three cores per case were counted. The cutoff was established using the 20 prostate tissue controls by counting 150 cells looking for the translocation. After samples were evaluated, the average and standard deviation of the nuclei with rearrangement were calculated. Then, a cutoff percentage was calculated as 3 positive standard deviations of the average percentage of rearrangement observed in normal tissues. Finally, this percentage was established in $15 \%$ of cells showing an altered FISH pattern.

2.5. TMPRSS2-ERG Detection by RT-PCR. One representative FFPE block was identified from each case and three sections of $20 \mu \mathrm{m}$ thick were obtained for total RNA extraction. The RNA quantification, RT-PCR, and the identification of TMPRSS2-ERG fusion transcripts were carried out as previously described in [18] (Figure 2).

PCR products of those TMPRSS2-ERG positive cases were purified, quantified, and sequenced on an ABI3130xl sequencer using the BigDye terminator v3.1 kit (Applied Biosystems, Inc., Foster City, CA) with specific primers. Sequencing Analysis v5.2 software (Applied Biosystems, Inc., Foster City, CA) and NCBI blast tool (http://www.ncbi .nlm.nih.gov/BLAST/ were used to confirm the sequences involved in the TMPRSS2-ERG fusions.

2.6. Performance Test. Considering FISH as gold standard, the sensitivity, specificity, positive predictive values (PPV) and negative predictive values (NPV) were calculated for RTPCR technique.

2.7. Statistical Analysis. Binary variables were used for the statistical analysis reflecting the positivity status of the measures. The association between TMPRSS2-ERG and clinicopathological parameters (categorical) was assessed using a chi-square test to determine homogeneity or linear trend for ordinal variables. The significance level was established at $5 \%$. The impact of the biological factors on biochemical (BPFS) and clinical progression free survival (PFS) was analyzed by Log-rank tests. Biochemical progression was defined as serum PSA level $>0.4 \mathrm{ng} / \mathrm{mL}$ during followup, whereas clinical progression was defined as local (prostatic fossa), regional (lymph nodes), or distant (metastasis). Univariate predictors of both BPFS and PFS were entered into a Cox proportional hazards model using stepwise selection to identify the independent predictors of poor outcome, with a confidence interval (CI) of 95\%. Statistical analysis was carried out using the SPSS statistical software package (version 15.0.1, SPSS Inc., Chicago, IL, USA).

\section{Results}

Since the three-color assay is a novel approach for TMPRSS2$E R G$ analysis, a break-apart FISH was first conducted in two TMAs including 38 cases in order to validate the tricolor Kreatech probes. Both approaches presented good concordance measured by chi-square test with a concordant rate of $80.6 \%$, a $13.8 \%$ false positive rate (FP), and $15.8 \%$ false negative rate $(P=0.013)$ taking break-apart assay as the gold standard.

For the whole series, using tricolor probes, 162 out of the $294 \mathrm{PCa}$ samples (55.1\%) were positive for genetic rearrangement. One hundred nineteen of these positive cases $(40.5 \%)$ showed a break-apart pattern and $35(11.9 \%)$ an interstitial deletion pattern. The remaining $8(2.7 \%)$ cases corresponded to undetermined fusion mechanisms. The presence of TMPRSS2-ERG rearrangement or the type of fusion mechanism assessed by means FISH showed no correlation with any of the clinicopathological parameters studied. There was only a trend towards statistical significance 


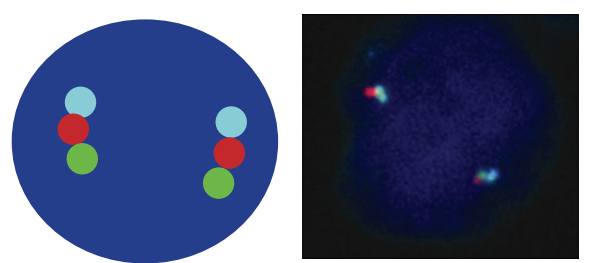

Normal

(A1)

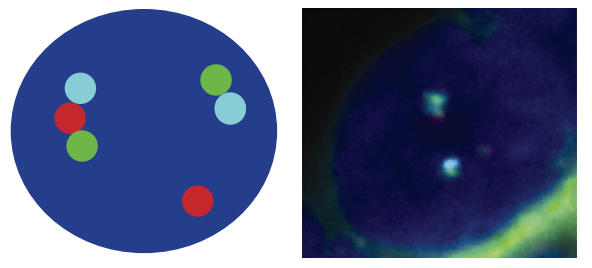

Balanced translocation

(A2)

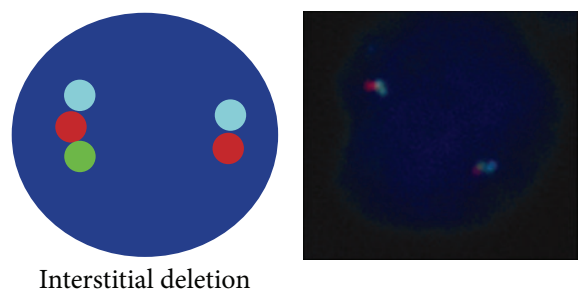

(A3)

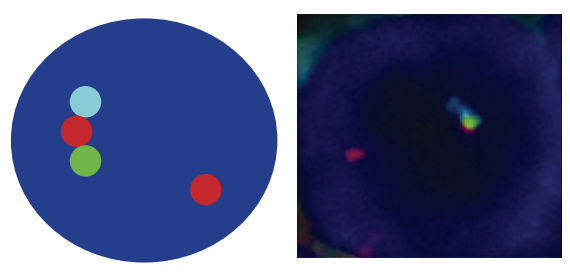

Undetermined rearrangement (1)

(A4)

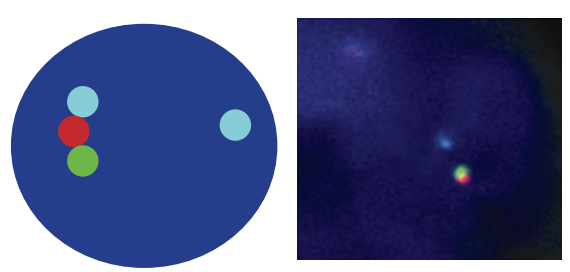

Undetermined rearrangement (2)

(A5)

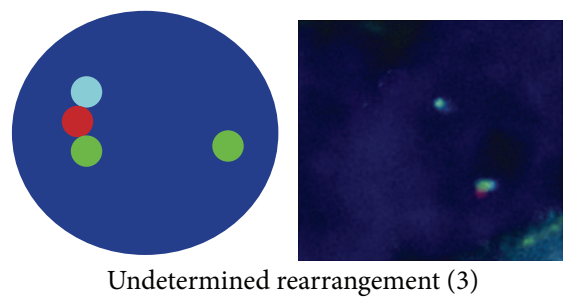

(A6)

(a)

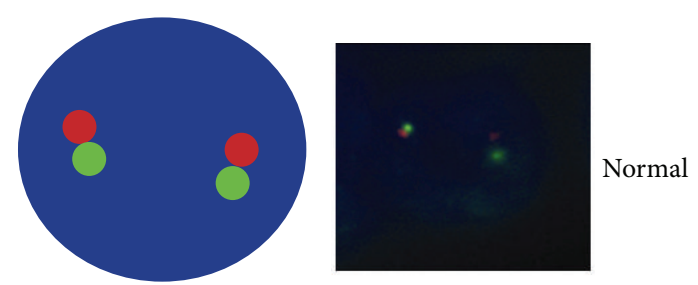

$(\mathrm{B} 1)$
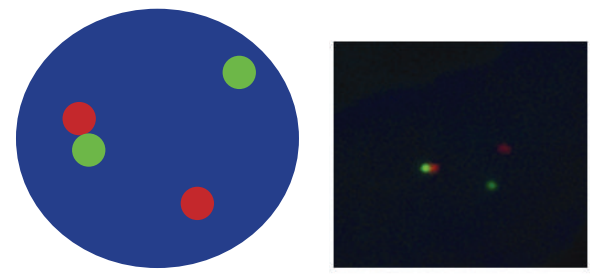

Balanced translocation

(B2)
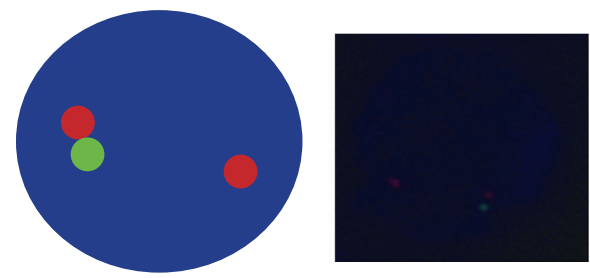

Interstitial deletion

(B3)

(b)

Figure 1: (a) Patterns obtained in the tricolor probe FISH analysis. Three fused signals indicate two normal alleles (A1); a balanced translocation pattern is considered with a fused (RGB) signal and an adjacent BG with a separated $R$ signals (A2); interstitial deletion is indicated by a RGB signal and a B and R adjacent signals (A3); one RGB signal plus only one R, B, or G signal (A4, A5, A6, resp.) corresponds to undetermined rearrangements. (b) Patterns of the dual color break-apart assay: normal pattern with two RG signals (B1); balanced translocation is indicated by a RG signal plus a G and R separated signals (B2); one RG signal plus a $R$ signal indicates interstitial deletion (B3). 


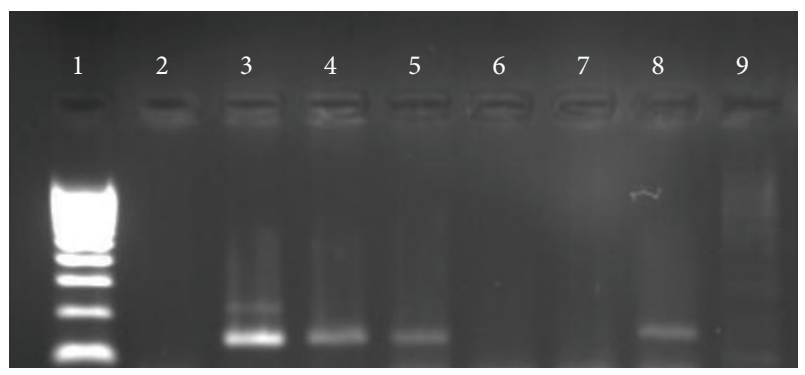

FIGURE 2: Gel electrophoresis showing representative results for the TMPRSS2-ERG gene status determined by RT-PCR. Lane 1: size marker; lines 3, 4, 5, and 8: positive cases showing a PCR product between the bands of 100 and 200 bp of the size marker; lanes 2, 6, and 7 are negative cases, and lane 9 is a negative template control.

between the presence of perineural invasion and the fusion gene detected by the tricolor FISH assay $(P=0.054)$ (Table 2).

With the RT-PCR approach, 152 out of the 294 (51.7\%) showed expression of the TMPRSS2-ERG fusion gene. One hundred and twenty-nine cases (43.9\%) harbored the T1E4 variant (TMPRSS2 exon 1 fused to the exon 4 of ERG), whereas $11(3.7 \%)$ expressed the T1E5 variant. The identification of fusion gene variant was not possible in two $(0.7 \%)$ cases.

Taking tricolor FISH as gold standard for determination of TMPRSS2-ERG, RT-PCR showed a sensitivity of $79.3 \%$ and a specificity of $81.9 \%$ with a Positive Predictive Value (PPV) of $80.9 \%$ and a Negative Predictive Value (NPV) of $80.2 \%$. FISH and RT-PCR assays showed concordant results in 237 out of 294 cases $(80.6 \%, P<0.001)$ (Table 3$)$.

Since FISH and RT-PCR showed a good agreement in the assessment of TMPRSS2-ERG status, we considered those cases as positive which presented the rearrangement determined by any of the two procedures. The presence of TMPRSS2-ERG according to this criterion showed no correlation with any of the clinical and pathological parameters (Table 4). However, and as aforementioned, there was a trend towards the statistical significance between the presence of TMPRSS2-ERG and perineural invasion $(P=0.091)$.

Regarding the prognostic implications of the presence of gene fusion, Table 5 shows the results of both the univariate and multivariate analyses of the clinicopathological and molecular parameters for both BPFS and PFS. Neither the TMPRSS2-ERG status nor the mechanisms of genetic rearrangement were related to the clinical outcome in our series (Figure 3).

\section{Discussion}

Currently, the standard diagnostic method for PCa is the pathological evaluation of prostate biopsy in patients with an elevated serum PSA level and/or an abnormal DRE. However, this clinical approach lacks sufficient sensitivity [19] being necessary for the discovery of new biomarkers that can improve the accuracy of PCa diagnosis. The objective of
TABLE 2: Correlation between the mechanism of the rearrangement and the clinical and pathological parameters.

\begin{tabular}{|c|c|c|c|c|}
\hline Parameters & $\begin{array}{c}\text { TMPRSS2-ERG } \\
\text { negative }(\%)\end{array}$ & $\begin{array}{c}\text { Break } \\
\text { apart } \\
(\%)\end{array}$ & $\begin{array}{c}\text { Interstitial } \\
\text { deletion (\%) }\end{array}$ & $P$ \\
\hline \multicolumn{5}{|l|}{ PSA } \\
\hline$<10 \mathrm{ng} / \mathrm{mL}$ & $85(47)$ & $73(73)$ & $23(23)$ & \multirow{3}{*}{0.907} \\
\hline $10-20 \mathrm{ng} / \mathrm{mL}$ & $29(45.3)$ & $29(45.3)$ & $6(9.4)$ & \\
\hline$>20 \mathrm{ng} / \mathrm{mL}$ & $18(43.9)$ & $17(41.4)$ & $6(14.6)$ & \\
\hline \multicolumn{5}{|l|}{ Gleason-sp } \\
\hline $2-6$ & $56(49.6)$ & $45(39.8)$ & $12(10.6)$ & \multirow{3}{*}{0.693} \\
\hline 7 & 57 (41.9) & $61(44.9)$ & $18(13.2)$ & \\
\hline$>7$ & $19(51.4)$ & $13(35.1)$ & $5(13.5)$ & \\
\hline \multicolumn{5}{|l|}{$\mathrm{cT}$} \\
\hline$\leq \mathrm{cT} 2 \mathrm{~b}$ & $122(45.9)$ & $109(41)$ & $23(23)$ & \multirow{2}{*}{0.251} \\
\hline$\geq \mathrm{cT} 3 \mathrm{a}$ & $9(50)$ & $9(50)$ & $0(0)$ & \\
\hline \multicolumn{5}{|l|}{$\mathrm{pT}$} \\
\hline$\leq \mathrm{pT} 2$ & $75(48.7)$ & $60(39)$ & $19(12.3)$ & \multirow{2}{*}{0.639} \\
\hline$\geq \mathrm{pT} 3$ & $57(43.5)$ & $58(44.3)$ & $16(12.2)$ & \\
\hline \multicolumn{5}{|l|}{$\mathrm{pN}^{*}$} \\
\hline pN0 & $84(45.4)$ & $75(40.5)$ & $26(14.1)$ & \multirow{2}{*}{0.177} \\
\hline $\mathrm{pN} \geq 1$ & $2(20)$ & $7(70)$ & $1(10)$ & \\
\hline \multicolumn{5}{|l|}{$\begin{array}{l}\text { Perineural } \\
\text { invasion }\end{array}$} \\
\hline Negative & $63(51.2)$ & $44(35.8)$ & $16(13)$ & 0.054 \\
\hline Positive & $53(38.1)$ & $70(50.4)$ & $16(11.5)$ & \\
\hline
\end{tabular}

TABLE 3: Crosstabs with the parameters of the comparison between RT-PCR and FISH techniques.

\begin{tabular}{lccc}
\hline & \multicolumn{2}{c}{ Fish } & $P$ \\
\hline RT-PCR & Negative & Positive & \\
Negative & $122(80.2 \%)$ & $27(19 \%)$ & $<0.001$ \\
Positive & $30(19.7 \%)$ & $115(81 \%)$ & \\
\hline
\end{tabular}

this study was to assess whether TMPRSS2-ERG fusion gene determined by tricolor FISH assay or RT-PCR could be used as part of the diagnostic panel of PCa.

Several strategies can be employed when a FISH experiment is designed. For instance, break-apart probes are widely validated in numerous studies constituting a valuable tool for determination of TMPRSS2-ERG in PCa $[4,20-$ 22]. However, this approach has its limitations. A positive result indicates that ERG is rearranged, but a second determination of TMPRSS2 would be necessary to confirm the presence of TMPRSS2-ERG because there are other genes that could be rearranged with ERG such as NDRG1 and $S L C 45 A 3[23,24]$. With a tricolor strategy one determination is enough to demonstrate the involvement of both genes. Furthermore, although the break-apart FISH has been widely used in investigation, this technique does not have diagnostic approval because noncommercial homemade probes are 
TABLE 4: Correlation between the presence of TMPRSS2-ERG determined by FISH and/or RT-PCR and the CPP.

\begin{tabular}{|c|c|c|c|}
\hline Parameters & $\begin{array}{c}\text { TMPRSS2-ERG } \\
\text { Negative (\%) }\end{array}$ & $\begin{array}{c}\text { TMPRSS2-ERG } \\
\text { Positive (\%) }\end{array}$ & $P$ \\
\hline \multicolumn{4}{|l|}{ PSA } \\
\hline$<10 \mathrm{ng} / \mathrm{mL}$ & $85(64.4)$ & $101(62.3)$ & \multirow{3}{*}{0.899} \\
\hline $10-20 \mathrm{ng} / \mathrm{mL}$ & $28(21.2)$ & $38(23.5)$ & \\
\hline$>20 \mathrm{ng} / \mathrm{mL}$ & $19(14.4)$ & $23(14.2)$ & \\
\hline \multicolumn{4}{|l|}{ Gleason-sp } \\
\hline $2-6$ & $50(37.9)$ & $68(42)$ & \multirow{3}{*}{0.292} \\
\hline 7 & $61(46.2)$ & $78(48.1)$ & \\
\hline$>7$ & $21(15.9)$ & $16(9.9)$ & \\
\hline \multicolumn{4}{|l|}{$\mathrm{cT}$} \\
\hline$\leq \mathrm{cT} 2 \mathrm{~b}$ & $123(93.2)$ & $150(93.8)$ & \multirow{2}{*}{0.514} \\
\hline$\geq \mathrm{cT} 3 \mathrm{a}$ & $9(6.8)$ & $10(6.3)$ & \\
\hline \multicolumn{4}{|l|}{$\mathrm{pT}$} \\
\hline$\leq \mathrm{pT} 2$ & $74(56.1)$ & $86(53.4)$ & \multirow{2}{*}{0.369} \\
\hline$\geq \mathrm{pT} 3$ & $58(43.9)$ & $75(46.6)$ & \\
\hline \multicolumn{4}{|l|}{$\mathrm{pN}^{*}$} \\
\hline $\mathrm{pN} 0$ & $82(96.5)$ & $108(93.9)$ & \multirow{2}{*}{0.317} \\
\hline $\mathrm{pN} \geq 1$ & $3(3.5)$ & $7(6.1)$ & \\
\hline \multicolumn{4}{|c|}{ Perineural invasion } \\
\hline Negative & $61(51.7)$ & $65(42.8)$ & \multirow[t]{2}{*}{0.091} \\
\hline Positive & $57(48.3)$ & $87(53.3)$ & \\
\hline
\end{tabular}

*Lymphadenectomy was limited to the obturator fossa in most of the cases.

used. However, the Kreatech Poseidon FISH probes have been developed specifically for their use in the clinical context constituting a valuable ancillary test in the PCa diagnosis.

In the series herein presented, the FISH analysis showed that $55.1 \%$ of PCa carried the TMPRSS2-ERG fusion gene of which $40.5 \%$ showed a split signal pattern and $11.9 \%$ evidenced an interstitial deletion. Remarkably, none of the analyzed cases showed the balanced translocation pattern consisting of one triplet signal (RGB) plus a B and G adjacent signals with a separated $\mathrm{R}$ signal as indicated by the commercial suppliers. However, the most frequent pattern was a triplet fused signal plus a BG signal (the pattern so-called break). This signal might correspond to a balanced translocation in which the $\mathrm{R}$ signal is not visible. However, it is also possible that the break pattern indicates an unknown mechanism of rearrangement between TMPRSS2 and ERG. In eight $(2.7 \%)$ cases harboring the fusion gene, it was not possible to determine the mechanism by which the rearrangement was produced. Seven of these cases were scored as TMPRSS2-ERG positive by RT-PCR, indicating a rearrangement between both genes. The frequency of the TMPRSS2-ERG is consistent with the series already published, ranging from $15 \%$ to $78 \%$ $[14,17,25,26]$. However, some differences might be explained by variations in the cohorts under study, low number of cases in some series, or sample selection bias $[17,25]$.

When referring to FISH, there are many questions associated with the interpretation of the results, for instance, the presence of multiple signals representing polyploidy or multiple copies of TMPRSS2-ERG difficult to interpret [27]; the number of nuclei that should be counted; and, the score of rearranged nuclei to be scored as positive [27, 28]. In our series, we evaluated a median of 150 nuclei per case and selected a $15 \%$ of rearranged nuclei as the optimum detection for TMPRSS2-ERG, and that discriminates between PCa and normal prostate tissue. This cutoff is similar to the one used by Machado et al. in the determination of EWSR1 in Ewing sarcoma by means of break-apart assay [27].

Unlike FISH, RT-PCR provides some advantages such as lower cost of the procedure and its capacity of discriminating different variants of the TMPRSS2-ERG fusion gene. In this regard, some authors have shown an association between some of these fusion subtypes with good [29] and poor prognoses [30]. However, because of its high sensitivity and cross-contamination, RT-PCR may on occasion give false positive results. Hence, RT-PCR is an interesting and useful technique in the diagnostic setting and should be considered as potential complement to FISH.

To our knowledge, there is only one study comparing FISH and RT-PCR in the determination of TMPRSS2-ERG in PCa showing a concordance of $84.7 \%$ [21], very similar to the herein reported. In the literature, there are other examples that compare FISH and RT-PCR in different settings $[27,31,32]$ : in breast cancer the comparison between both techniques in the determination the HER2 status has shown a good concordance ranging between $80-97 \%$ [33-35]; in the case of dermatofibrosarcoma protuberans, this concordance is of $67 \%$ for detection of COL1A1-PDGFB rearrangement [28]; and finally, in Ewing Sarcomas, several studies showed concordances between 55.5 and 100\% [31, 36-38].

Regarding the association with clinical and pathological parameters, no correlation between the rearrangement mechanisms and the surrogate prognostic parameters was found. A trend towards statistical significance was observed between the presence of TMPRSS2-ERG and a higher perineural invasion, which is a surrogate parameter of poor prognosis, although as an individual variable it loses its importance as a prognostic value in most of multivariate analyses.

One important question to be addressed in this context is the prognostic implications of the presence of fusion gene in PCa samples. To date, it remains controversial, and there are many studies defending the relationship between TMPRSS2-ERG and both good $[29,39,40]$ and poor [12, $17,30,41]$ prognoses. In addition, other many authors find no correlation between the presence of TMPRSS2-ERG and PCa outcome [42]. Interestingly, Attard et al. reported that the presence of TMPRSS2-ERG resulted from an interstitial deletion accompanied by a high copy number of this gene (the so called class 2+ Edel), with a poor prognosis [12], but to date, there are no studies confirming these observations. A recent work of Tomlins et al. reported a prognostic panel composed by TMPRSS2-ERG and PCA3 measured in urine samples by means of transcription-mediated amplification [43]. In addition, our group reported the different prognostic panel in PCa that harbors the fusion gene indicating that the determination of this biomarker would be useful in 


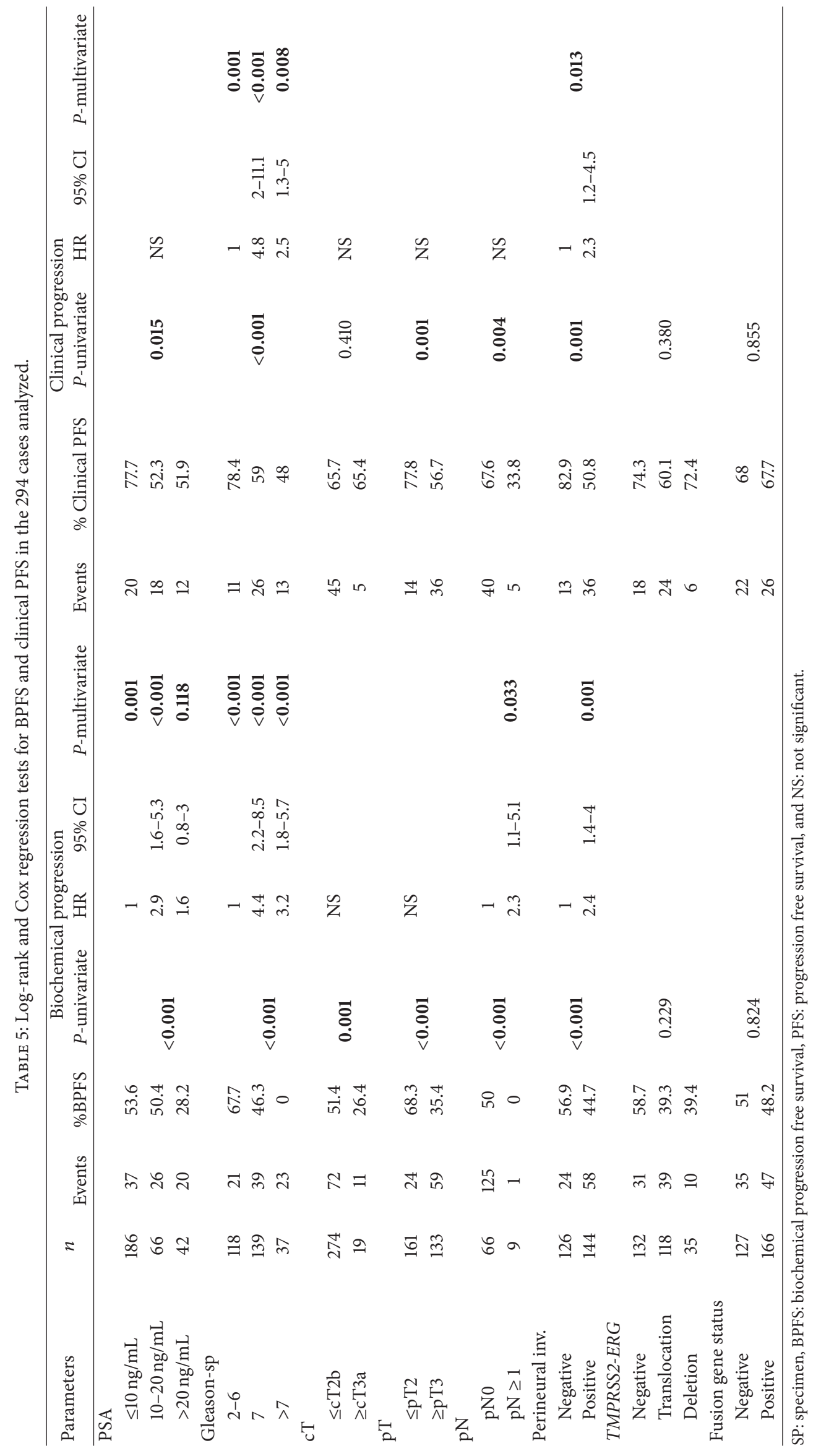




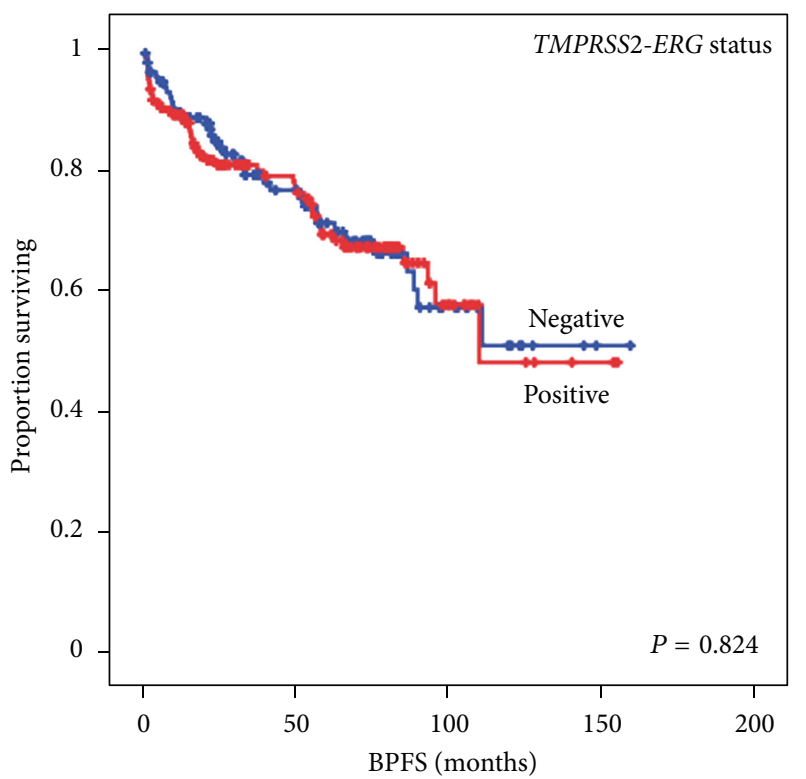

(a)

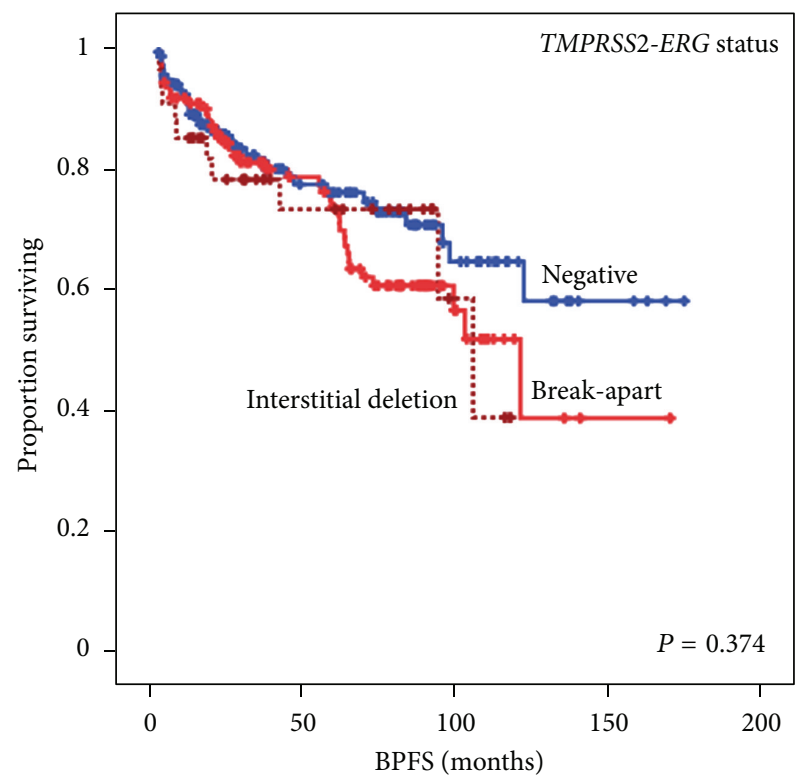

(b)

FIGURE 3: Kaplan-Meier plots of the univariate survival analysis. (a) Biochemical progression free survival (BPFS) according to the TMPRSS2ERG status measured by FISH and/or RT-PCR. (b) BPFS according to the mechanism of the rearrangement determined by tricolor FISH assay.

distinguishing groups of PCa patients defined by differential prognostic indicators [18].

Despite the great number of studies published on this matter, and due to differences with regard to the technology used to determine TMPRSS2-ERG status and the heterogeneity of the cohorts, it is difficult to draw conclusions about the prognostic involvement of this biomarker. Herein we have analyzed a well-defined cohort with a long followup in order to avoid these problems and no differences between the TMPRSS2-ERG status and the genetic mechanisms by which it has been produced with the prognosis of patients with $\mathrm{PCa}$ have been found.

In summary, we have demonstrated the usefulness of a commercial tricolor probe FISH approach for the identification for the TMPRSS2-ERG status and the genetic mechanism responsible of this fusion gene, as well as high grade of concordance between this strategy and RT-PCR. Furthermore, we have proved that the TMPRSS2-ERG status, although specific for $\mathrm{PCa}$, is not valid as a prognostic biomarker in $\mathrm{PCa}$ patients treated with radical prostatectomy.

\section{Authors' Contribution}

A. Fernández-Serra and L. Rubio contributed equally to the paper.

\section{Acknowledgments}

The authors thank Ana Cervera Ferri for drawing the paper and acknowledge the technical assistance of María García Flores, Tania Mazcuñán Vitiello, and Patricia Carretero Hinojosa. They also thank the Biobank of the Fundación
Instituto Valenciano de Oncología for providing the biological samples for the analysis. This study has been funded by the Grants FIS PI06/01619 and PI10/01206 from the Instituto de Salud Carlos III, Madrid, ACOMP 12/029 from the Generalitat Valenciana, Valencia, and Astra Zeneca, Spain.

\section{References}

[1] J. H. Hayes, D. A. Ollendorf, S. D. Pearson et al., "Active surveillance compared with initial treatment for men with lowrisk prostate cancer: a decision analysis," Journal of the American Medical Association, vol. 304, no. 21, pp. 2373-2380, 2010.

[2] A. H. Thomson, S. Kulkarni, and A. Bahl, "Primary cryotherapy with salvage external beam radiotherapy for locally recurrent prostate cancer," Clinical Oncology, vol. 20, no. 5, p. 385, 2008.

[3] F. Rabbani, N. Stroumbakis, B. R. Kava, M. S. Cookson, and W. R. Fair, "Incidence and clinical significance of false-negative sextant prostate biopsies," The Journal of Urology, vol. 159, no. 4, pp. 1247-1250, 1998.

[4] S. A. Tomlins, D. R. Rhodes, S. Perner et al., "Recurrent fusion of TMPRSS2 and ETS transcription factor genes in prostate cancer," Science, vol. 310, no. 5748, pp. 644-648, 2005.

[5] S. A. Tomlins, R. Mehra, D. R. Rhodes et al., "TMPRSS2:ETV4 gene fusions define a third molecular subtype of prostate cancer," Cancer Research, vol. 66, no. 7, pp. 3396-3400, 2006.

[6] S. A. Narod, A. Seth, and R. Nam, "Fusion in the ETS gene family and prostate cancer," British Journal of Cancer, vol. 99, no. 6, pp. 847-851, 2008.

[7] S. A. Tomlins, B. Laxman, S. Varambally et al., "Role of the TMPRSS2-ERG gene fusion in prostate cancer," Neoplasia, vol. 10, no. 2, pp. 177-188, 2008.

[8] S. A. Tomlins, A. Bjartell, A. M. Chinnaiyan et al., "ETS gene fusions in prostate cancer: from discovery to daily clinical practice," European Urology, vol. 56, no. 2, pp. 275-286, 2009. 
[9] A. Fernández-Serra, J. Rubio-Briones, Z. García-Casado, E. Solsona, and J. A. López-Guerrero, "Prostate cancer: the revolution of the fusion genes," Actas Urologicas Espanolas, vol. 35, no. 7, pp. 420-428, 2011.

[10] Q. P. Sun, L. Y. Li, Z. Chen et al., "Detection of TMPRSS2-ETS fusions by a multiprobe fluorescence in situ hybridization assay for the early diagnosis of prostate cancer: a pilot study," Journal of Molecular Diagnostics, vol. 12, no. 5, pp. 718-724, 2010.

[11] M. Yoshimoto, J. C. Cutz, P. A. S. Nuin et al., "Interphase FISH analysis of PTEN in histologic sections shows genomic deletions in $68 \%$ of primary prostate cancer and $23 \%$ of highgrade prostatic intra-epithelial neoplasias," Cancer Genetics and Cytogenetics, vol. 169, no. 2, pp. 128-137, 2006.

[12] G. Attard, J. Clark, L. Ambroisine et al., "Duplication of the fusion of TMPRSS2 to ERG sequences identifies fatal human prostate cancer," Oncogene, vol. 27, no. 3, pp. 253-263, 2008.

[13] G. Attard, J. Clark, L. Ambroisine et al., "Heterogeneity and clinical significance of ETV1 translocations in human prostate cancer," British Journal of Cancer, vol. 99, no. 2, pp. 314-320, 2008.

[14] S. Perner, F. Demichelis, R. Beroukhim et al., "TMPRSS2:ERG fusion-associated deletions provide insight into the heterogeneity of prostate cancer," Cancer Research, vol. 66, no. 17, pp. 83378341, 2006.

[15] B. Lu, B. Maqsodi, W. Yang et al., "Detection of TMPRSS2-ERG fusion gene expression in prostate cancer specimens by a novel assay using branched DNA," Urology, vol. 74, no. 5, pp. 11561161, 2009.

[16] M. Yoshimoto, A. M. Joshua, S. Chilton-MacNeill et al., "Threecolor FISH analysis of TMPRSS2/ERG fusions in prostate cancer indicates that genomic microdeletion of chromosome 21 is associated with rearrangement," Neoplasia, vol. 8, no. 6, pp. 465469, 2006.

[17] F. Demichelis, K. Fall, S. Perner et al., "TMPRSS2:ERG gene fusion associated with lethal prostate cancer in a watchful waiting cohort," Oncogene, vol. 26, no. 31, pp. 4596-4599, 2007.

[18] J. Rubio-Briones, A. Fernández-Serra, A. Calatrava et al., "Clinical implications of TMPRSS2-ERG gene fusion expression in patients with prostate cancer treated with radical prostatectomy," The Journal of Urology, vol. 183, no. 5, pp. 2054-2061, 2010.

[19] B. Djavan, M. Remzi, C. C. Schulman, M. Marberger, and A. R. Zlotta, "Repeat prostate biopsy: who, how and when? A review," European Urology, vol. 42, no. 2, pp. 93-103, 2002.

[20] J. M. Mosquera, S. Perner, E. M. Genega et al., "Characterization of TMPRSS2-ERG fusion high-grade prostatic intraepithelial neoplasia and potential clinical implications," Clinical Cancer Research, vol. 14, no. 11, pp. 3380-3385, 2008.

[21] J. J. Tu, S. Rohan, J. Kao, N. Kitabayashi, S. Mathew, and Y. T. Chen, "Gene fusions between TMPRSS2 and ETS family genes in prostate cancer: frequency and transcript variant analysis by RT-PCR and FISH on paraffin-embedded tissues," Modern Pathology, vol. 20, no. 9, pp. 921-928, 2007.

[22] M. Yoshimoto, A. M. Joshua, I. W. Cunha et al., "Absence of TMPRSS2:ERG fusions and PTEN losses in prostate cancer is associated with a favorable outcome," Modern Pathology, vol. 21, no. 12, pp. 1451-1460, 2008.

[23] D. Pflueger, D. S. Rickman, A. Sboner et al., "N-myc downstream regulated gene 1 (NDRG1) is fused to $E R G$ in prostate cancer," Neoplasia, vol. 11, no. 8, pp. 804-811, 2009.

[24] B. Han, R. Mehra, S. M. Dhanasekaran et al., "A fluorescence in situ hybridization screen for E26 transformation-specific aberrations: identification of DDX5-ETV4 fusion protein in prostate cancer," Cancer Research, vol. 68, no. 18, pp. 7629-7637, 2008.

[25] M. J. Soller, M. Isaksson, P. Elfving, W. Soller, R. Lundgren, and I. Panagopoulos, "Confirmation of the high frequency of the TMPRSS2/ERG fusion gene in prostate cancer," Genes Chromosomes and Cancer, vol. 45, no. 7, pp. 717-719, 2006.

[26] S. A. Tomlins, R. Mehra, D. R. Rhodes et al., "Integrative molecular concept modeling of prostate cancer progression," Nature Genetics, vol. 39, no. 1, pp. 41-51, 2007.

[27] I. Machado, R. Noguera, A. Pellin et al., "Molecular diagnosis of ewing sarcoma family of tumors: a comparative analysis of 560 cases with fish and rt-pcr," Diagnostic Molecular Pathology, vol. 18, no. 4, pp. 189-199, 2009.

[28] R. Salgado, B. Llombart, R. M. Pujol et al., "Molecular diagnosis of dermatofibrosarcoma protuberans: a comparison between reverse transcriptase-polymerase chain reaction and fluorescence in situ hybridization methodologies," Genes Chromosomes and Cancer, vol. 50, no. 7, pp. 510-517, 2011.

[29] K. G. Hermans, J. L. Boormans, D. Gasi et al., "Overexpression of prostate-specific TMPRSS2(exon 0)-ERG fusion transcripts corresponds with favorable prognosis of prostate cancer," Clinical Cancer Research, vol. 15, no. 20, pp. 6398-6403, 2009.

[30] J. Wang, Y. Cai, C. Ren, and M. Ittmann, "Expression of variant TMPRSS2/ERG fusion messenger RNAs is associated with aggressive prostate cancer," Cancer Research, vol. 66, no. 17, pp. 8347-8351, 2006.

[31] R. S. Bridge, V. Rajaram, L. P. Dehner, J. D. Pfeifer, and A. Perry, "Molecular diagnosis of Ewing sarcoma/primitive neuroectodermal tumor in routinely processed tissue: a comparison of two FISH strategies and RT-PCR in malignant round cell tumors," Modern Pathology, vol. 19, no. 1, pp. 1-8, 2006.

[32] T. B. Lewis, C. M. Coffin, and P. S. Bernard, "Differentiating Ewing's sarcoma from other round blue cell tumors using a RTPCR translocation panel on formalin-fixed paraffin-embedded tissues," Modern Pathology, vol. 20, no. 3, pp. 397-404, 2007.

[33] M. L. Wallander, K. B. Geiersbach, S. R. Tripp, and L. J. Layfield, "Comparison of reverse transcription-polymerase chain reaction, immunohistochemistry, and fluorescence in situ hybridization methodologies for detection of echinoderm microtubule-associated proteinlike 4-anaplastic lymphoma kinase fusion-positive non-small cell lung carcinoma: implications for optimal clinical testing," Archives of Pathology \& Laboratory Medicine, vol. 136, no. 7, pp. 796-803, 2012.

[34] L. Dvorak, M. Dolan, J. Fink, L. Varghese, J. Henriksen, and H. E. Gulbahce, "Correlation between HER2 determined by fluorescence in situ hybridization and reverse transcriptionpolymerase chain reaction of the oncotype DX test," Applied Immunohistochemistry \& Molecular Morphology, vol. 21, no. 3, pp. 196-199, 2013.

[35] F. L. Baehner, N. Achacoso, T. Maddala et al., "Human epidermal growth factor receptor 2 assessment in a case-control study: comparison of fluorescence in situ hybridization and quantitative reverse transcription polymerase chain reaction performed by central laboratories," Journal of Clinical Oncology, vol. 28, no. 28, pp. 4300-4306, 2010.

[36] A. L. Folpe, J. R. Goldblum, B. P. Rubin et al., "Morphologic and immunophenotypic diversity in Ewing family tumors: a study of 66 genetically confirmed cases," American Journal of Surgical Pathology, vol. 29, no. 8, pp. 1025-1033, 2005. 
[37] S. Kumar, S. Pack, D. Kumar et al., "Detection of EWS-FLI-1 fusion in Ewing's sarcoma/peripheral primitive neuroectodermal tumor by fluorescence in situ hybridization using formalinfixed paraffin-embedded tissue," Human Pathology, vol. 30, no. 3, pp. 324-330, 1999.

[38] M. K. Fritsch, J. A. Bridge, A. E. Schuster, E. J. Perlman, and P. Argani, "Performance characteristics of a reverse transcriptasepolymerase chain reaction assay for the detection of tumorspecific fusion transcripts from archival tissue," Pediatric and Developmental Pathology, vol. 6, no. 1, pp. 43-53, 2003.

[39] O. R. Saramäki, A. E. Harjula, P. M. Martikainen, R. L. Vessella, T. L. J. Tammela, and T. Visakorpi, "TMPRSS2.ERG fusion identifies a subgroup of prostate cancers with a favorable prognosis," Clinical Cancer Research, vol. 14, no. 11, pp. 33953400, 2008.

[40] G. Petrovics, A. Liu, S. Shaheduzzaman et al., "Frequent overexpression of ETS-related gene-1 (ERG1) in prostate cancer transcriptome," Oncogene, vol. 24, no. 23, pp. 3847-3852, 2005.

[41] A. B. Rajput, M. A. Miller, A. de Luca et al., "Frequency of the TMPRSS2:ERG gene fusion is increased in moderate to poorly differentiated prostate cancers," Journal of Clinical Pathology, vol. 60, no. 11, pp. 1238-1243, 2007.

[42] A. Gopalan, M. A. Leversha, J. M. Satagopan et al., "TMPRSS2$E R G$ gene fusion is not associated with outcome in patients treated by prostatectomy," Cancer Research, vol. 69, no. 4, pp. 1400-1406, 2009.

[43] S. A. Tomlins, S. M. Aubin, J. Siddiqui et al., "Urine TMPRSS2:ERG fusion transcript stratifies prostate cancer risk in men with elevated serum PSA," Science Translational Medicine, vol. 3, no. 94, Article ID 94ra72, 2011. 


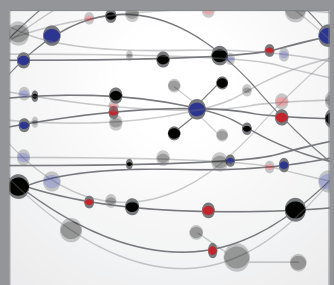

The Scientific World Journal
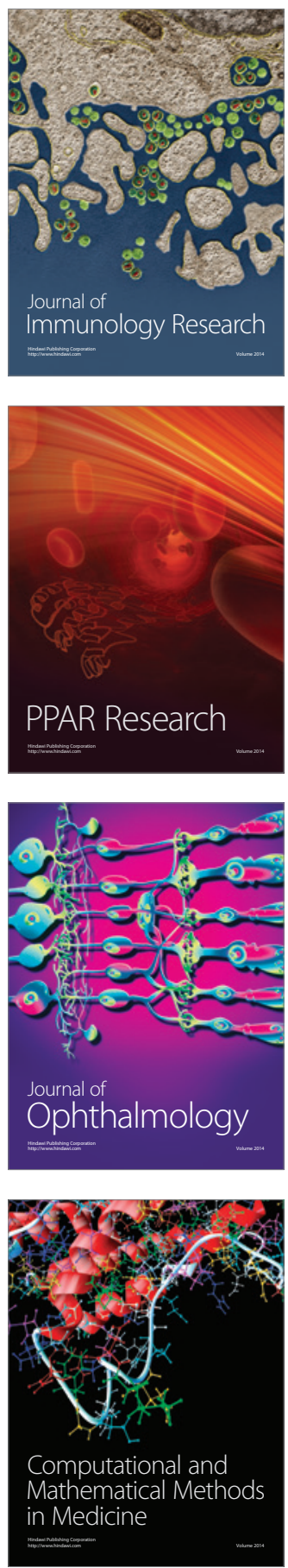

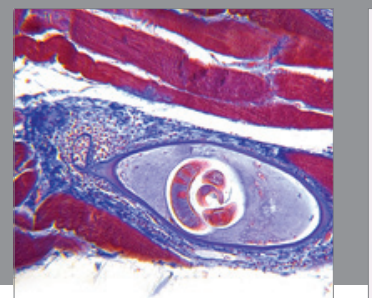

Gastroenterology

Research and Practice
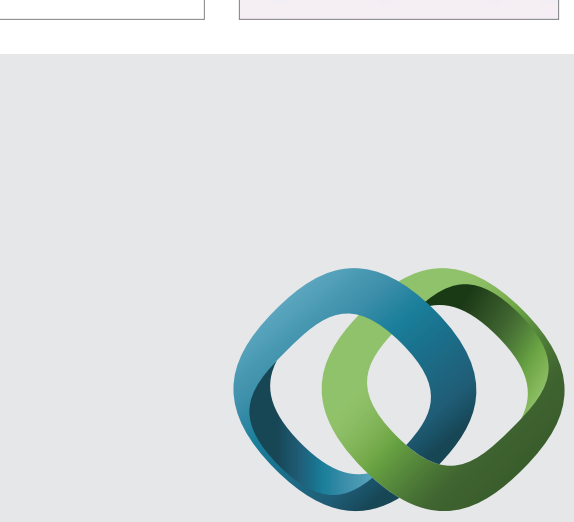

\section{Hindawi}

Submit your manuscripts at

http://www.hindawi.com
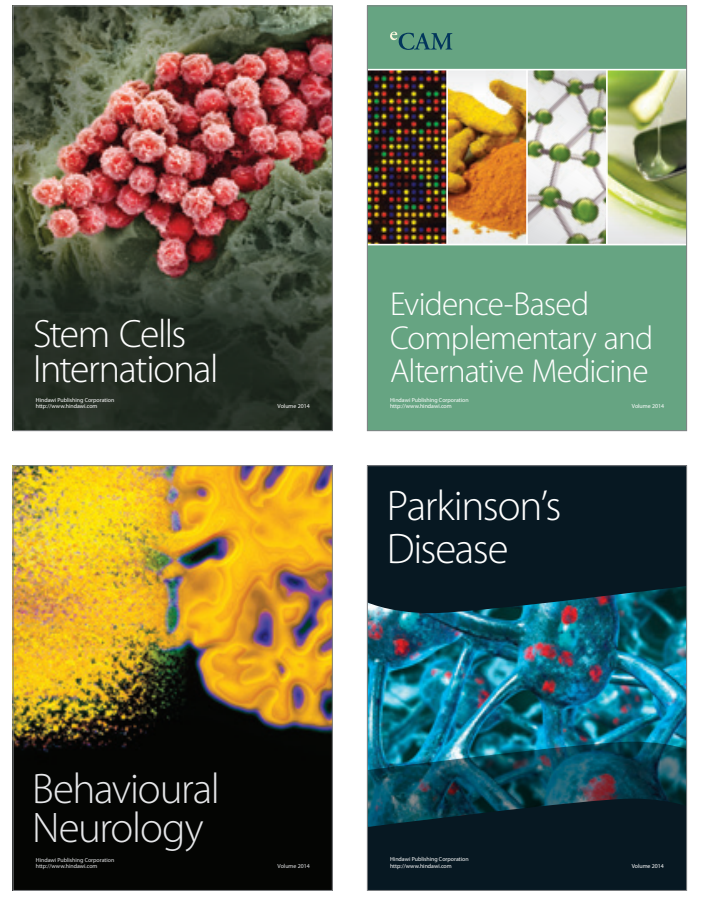
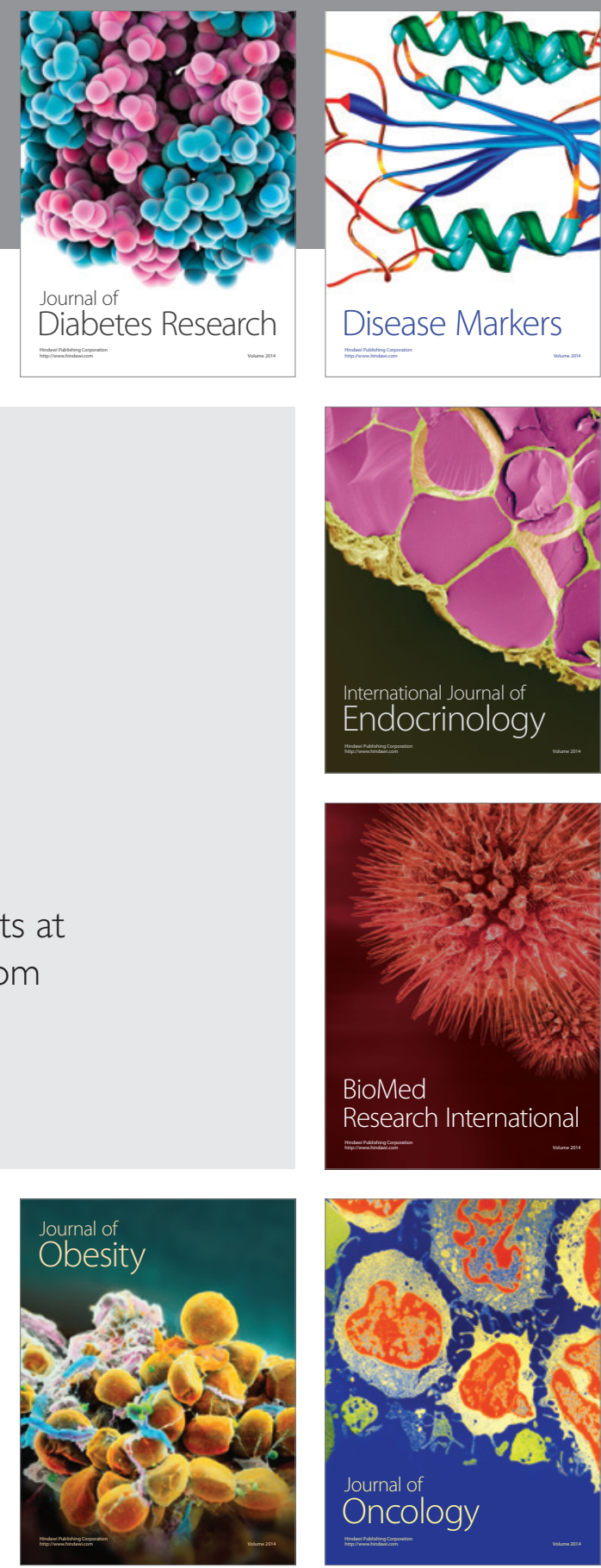

Disease Markers
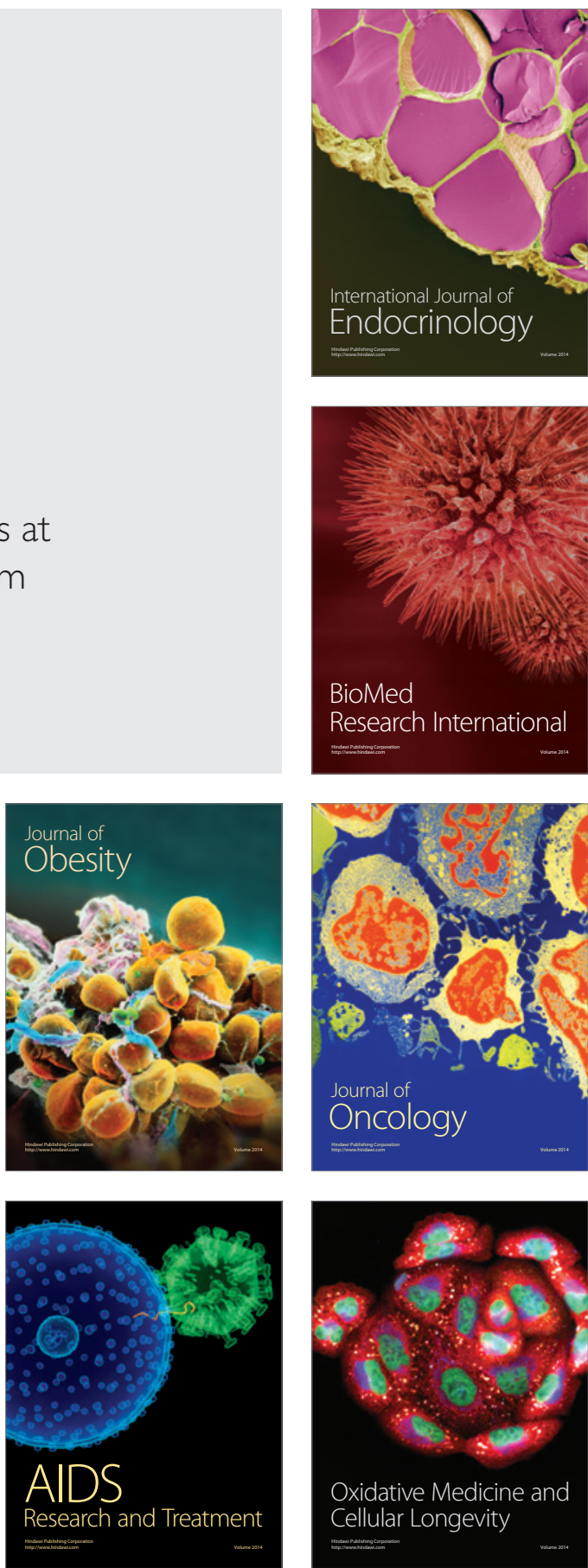